\title{
EXTREMAL PROBLEMS AND HARMONIC INTERPOLATION ON OPEN RIEMANN SURFACES $\left({ }^{1}\right)$
}

\author{
BY \\ LEO SARIO
}

\section{INTRODUCTION}

1. The ultimate purpose of the present paper is to study interpolation of harmonic and analytic functions on open Riemann surfaces $W$. We shall, however, first take a less restricted viewpoint and consider, in general, extremal problems on Riemann surfaces.

Extrema of given functionals can often be immediately found in the special case where $W$ is compact and bounded by a finite number of analytic Jordan curves. The difficulties arise when the contours are arbitrary or, as is always the case for an infinite genus, the boundary exists in an ideal sense only. With this in view, we shall start with a theorem for reducing extremal problems from the general case of an open Riemann surface $W$ to the special case just mentioned. The content of the reduction theorem (no. 5) will be, roughly speaking, as follows:

If the functional to be minimized increases with the region, and if the minimizing functions on compact subregions $W_{n}$ with analytic boundaries form a normal family, then the solvability of the extremal problem for $W_{n}$ implies that for $W$.

2. The reduction theorem will enable us to establish a rather general theorem on minimizing functionals of harmonic functions on Riemann surfaces. We consider the class of single-valued harmonic functions $p$ with a finite number of prescribed poles or logarithmic singularities $\zeta_{i}(i=1, \cdots, n)$ on $W$. The expansions of $p$ about $\zeta_{i}$ have the form

$$
p=\operatorname{Re}\left\{\sum_{0}^{\infty} a_{\nu} z^{\nu}+\lambda\left(\sum_{1}^{m} b_{\nu} z^{-\nu}-c \log |z|\right)\right\},
$$

where $a_{v}, b_{v}$ are complex, $c$ real coefficients, $\lambda$ a real parameter, and $z$ the local uniformizer. The following combination of the coefficients will be instrumental:

$$
\mu=2 \pi \sum_{i=1}^{n} \operatorname{Re}\left[c a_{0}+\sum_{\nu=1}^{m} \nu b_{\nu} a_{v}\right] .
$$

For fixed $b_{v}, c$ and subsequently fixed $\lambda$, the class $\{p\}$ shall be designated

Received by the editors July 5, 1954.

(1) Sponsored by the Office of Ordnance Research, U. S. Army, Contract DA 19-020-ORD1903, Harvard University. Presented at the Conference on Functions of a Complex Variable, Ann Arbor, on June 30, 1953. 
by $C_{\lambda}$. We set $\lambda=h+k$, with real $h, k$, and choose

$$
m(p)=\int_{\beta} p d p^{*}+(k-h) \mu
$$

for the functional to be minimized. Here the asterisk denotes the harmonic conjugate, $\beta$ stands for the ideal boundary, and the integral is understood as the limit of integrals along the boundaries of exhausting subregions. The theorem to be established (no. 7) follows:

There exists a unique function $p_{h k}$ in $C_{\lambda}$ which minimizes $m(p)$. For varying $h, k$, the $p_{h k}$ are interrelated by

$$
p_{h k}=h p_{0}+k p_{1},
$$

where $p_{0}, p_{1}$ stand for $p_{1,0}, p_{0,1}$, respectively. The minimum value of $m(p)$ is $k^{2} \mu_{1}-h^{2} \mu_{0}$, with $\mu_{0}=\mu\left(p_{0}\right), \mu_{1}=\mu\left(p_{1}\right)$, and the deviation from this minimum is given by the Dirichlet integral of $p-p_{h k}$ :

$$
m(p)=k^{2} \mu_{1}-h^{2} \mu_{0}+D\left(p-p_{h k}\right) .
$$

It is of interest that here $p_{0}, p_{1}$ are the principal functions $[16 ; 18]$ which correspond to the normal linear operators $L_{0}, L_{1}$, characterized by vanishing normal derivatives and constant boundary values, respectively. By fixing $b_{\nu}, c, \lambda$, we can choose the class $C_{\lambda}$ of functions (1); by subsequently fixing $h, k$ within $h+k=\lambda$, we can select the functional (3) to be minimized in $C_{\lambda}$. Thus the linear combinations $h p_{0}+k p_{1}$ of the principal functions furnish the solutions to extremal problems of great variety and generality. A detailed account of these applications will be given in [19]. In the present paper we shall make use of the theorem in interpolation problems.

3 . Let $u_{i \nu}(i=1, \cdots, n ; \nu=0, \cdots, m)$ be arbitrary real constants. Consider regular single-valued harmonic functions $u(z)$ on $W$ with

$$
\partial^{v} u\left(\zeta_{i}\right) / \partial x^{\nu}=u_{i v}
$$

Here $z=x+i y$ is the local uniformizer at $\zeta_{i}$. We are interested in the interpolation problem of constructing a harmonic function satisfying (6) and with a smallest possible value of the Dirichlet integral. If $m=0$, we are dealing with the simplest case where the $u\left(\zeta_{i}\right)$ only are given. If $n=1$, we have functions with values and derivatives, up to the $m$ th order, prescribed at one point only.

We shall show (no. 24) that the principal functions $p_{0}, p_{1}$ again provide us with the solution:

The function

$$
u_{0}=p_{0}-p_{1},
$$

for properly chosen $b_{v}, c$, satisfies the conditions (6) and minimizes $D(u)$. The value of the minimum is $\mu_{0}-\mu_{1}$, and the deviation from this minimum is given 
by the Dirichlet integral of $u-u_{0}$ :

$$
D(u)=\mu_{0}-\mu_{1}+D\left(u-u_{0}\right) .
$$

The possibility of a proper choice of the $b_{\nu}, c$ will be fully characterized by the nonvanishing of a determinant (no. 23).

If $W$ is planar, we shall also consider single-valued analytic functions $V(z)$ with prescribed values of $V^{(\nu)}\left(\zeta_{i}\right), \nu=1, \cdots, m$. Then the analytic completions of suitably modified functions $p_{0}, p_{1}$ will furnish the solution, which exists if and only if the boundary of $W$ is not $A D$-removable (no. 28).

With regard to the vast literature concerning interpolation problems in the unit circle, we refer here to the well-known results of Walsh [21], Pick $[15]$, Nevanlinna $[13 ; 14]$ and Weyl $[22]$. These authors discussed the interpolation problem from the viewpoint of minimizing mean values. The question of minimizing the Dirichlet integral was first treated by Kubota $[6 ; 7]$, Takenaka [20] and, for more general plane regions, by Lokki [9-12], Garabedian and Schiffer [4], and Lehto [8]. The prescribed quantities in these investigations were the values of the functions $(m=0)$, while Bergman [3] also considered values of derivatives at a fixed point $(n=1)$. Concerning extremal problems for differentials, the reader is referred to the comprehensive study [1] by Ahlfors.

In what follows, any finite values of $n, m$ will be permitted and the region of existence is allowed to be an arbitrary open Riemann surface. Our approach, sketched above, is based on the extremal method introduced in [17].

\section{\$1. REDUCTION THEOREM}

4. Let $W$ be an arbitrary open Riemann surface and $C$ a class of functions on $W$. Consider an exhaustion $\left\{W_{n}\right\}$ of $W$ by compact subregions $W_{n}$, each bounded by a finite set $\beta_{n}$ of analytic Jordan curves. On $W_{n}$, a class $C_{n}$ of functions is given with the property that the restriction to $W_{n-1}$ of $p \in C_{n}$ belongs to $C_{n-1}$. It is understood that this holds, correspondingly, for $W, W_{n}$ and $C, C_{n}$.

For $W_{n}$ and $p \in C_{n}$, a functional $m\left(W_{n}, p\right)$ is given, such that, for $p^{\prime} \in C_{n}$ tending uniformly on $W_{n}$ to $p^{\prime \prime} \in C_{n}$, we have $m\left(W_{n}, p^{\prime}\right) \rightarrow m\left(W_{n}, p^{\prime \prime}\right)$. The functional $m(W, p)$ for $W$ and $p \in C$ is defined by

$$
m(W, p)=\lim _{n \rightarrow \infty} m\left(W_{n}, p\right),
$$

the existence of the limit being postulated.

The problem is to find a function $p_{W}$ which minimizes $m(W, p)$ in $C$, and to determine properties of $p_{W}$ and $m\left(W, p_{W}\right)$.

5. We shall establish the following

REDUCTION THEOREM. Suppose that the functionals $m\left(W_{n}, p\right)$ defined above satisfy the following conditions: 
$1^{\circ}$. The problem has a solution for $W_{n}$, that is, there exists a function $p_{n}$ in $C_{n}$ with

$$
\min _{p \in C_{n}} m\left(W_{n}, p\right)=m\left(W_{n}, p_{n}\right) .
$$

$2^{\circ}$. The functional $m$ is an increasing function of the region:

$$
m\left(W_{n}, p\right) \leqq m\left(W_{n+1}, p\right)
$$

for $p \in C_{n+1}$.

$3^{\circ}$. The family $\left\{p_{n}\right\}$ is normal, the limiting functions on $W$ belonging to $C$.

Then every limiting function, $p_{W}=\lim _{n \rightarrow \infty} p_{n}$, say, is a solation of the extremal problem for $W$,

$$
\min _{p \in c} m(W, p)=m\left(W, p_{W}\right)
$$

and the value of the minimum is

$$
m\left(W, p_{W}\right)=\lim _{n \rightarrow \infty} m\left(W_{n}, p_{n}\right) .
$$

Proof. We first observe that, by definition,

$$
m\left(W, p_{W}\right)=\lim _{n \rightarrow \infty} m\left(W_{n}, p_{W}\right)=\lim _{n \rightarrow \infty} \lim _{k \rightarrow \infty} m\left(W_{n}, p_{k}\right) .
$$

Consequently, by virtue of condition $2^{\circ}$,

$$
m\left(W, p_{W}\right) \leqq \lim _{n \rightarrow \infty} m\left(W_{n}, p_{n}\right) .
$$

On the other hand, we have for $p \in C$ the inequalities

$$
m\left(W_{n}, p_{n}\right) \leqq m\left(W_{n}, p\right) \leqq m(W, p) .
$$

As this holds for each $W_{n}$ and $p \in C$, we deduce that

$$
\lim _{n \rightarrow \infty} m\left(W_{n}, p_{n}\right) \leqq \inf m(W, p) \leqq m\left(W, p_{W}\right) .
$$

The statements (4) and (5) now follow on combining (6) and (7).

\section{§2. A theOREM ON Minima of FUnCtionals}

6. We consider the class of single-valued harmonic functions $p$ with a finite number of given singularities on an open Riemann surface $W$. It is no restriction to assume that the singularities $\zeta_{i}(i=1, \cdots, n)$ are centers of disjoint parametric disks $K_{i}$. The functions $p$ have the following expansions in $K_{i}$ :

$$
p=\operatorname{Re}\left\{\sum_{0}^{\infty} a_{\nu} z^{\nu}+\lambda \cdot\left(\sum_{1}^{m} b_{\nu} z^{-\nu}-c \log |z|\right)\right\}
$$


Here $a_{v}, b_{\nu}$ are complex, $c$ real coefficients, and $\lambda$ a real parameter. The class of functions (1) with fixed $b_{\nu}, c$ and subsequently fixed $\lambda$ shall be denoted by $C_{\lambda}$. In particular, $C_{0}$ is the class of all single-valued regular harmonic functions $p$ on $W$.

For simplicity, we shall not use additional indices $i$ to distinguish the $a_{v}, b_{v}, c, m$ and $z$ corresponding to the various $K_{i}$. To avoid unnecessary constants, we also shall assume that the functions $p$ have been normalized, by adding suitable real constants, so as to have $\operatorname{Re}\left\{a_{0}\right\}=0$ at $\zeta_{1}$. Moreover, the coefficients $c$, whose reality is implied by the single-valuedness of $p$, are supposed to satisfy the condition $\sum_{i=1}^{n} c=0$. We shall use the abbreviation

$$
\mu=2 \pi \sum_{i=1}^{n} \operatorname{Re}\left[c a_{0}+\sum_{\nu=1}^{m} \nu b_{\nu} a_{\nu}\right] .
$$

7. Set $\lambda=h+k$ with real $h, k$, and consider the following functional of $p$ :

$$
m(W, p)=\int_{\beta} p d p^{*}+(k-h) \mu .
$$

Here the integral along the ideal boundary $\beta$ of $W$ is defined as the limit of integrals taken along boundaries of exhausting regions.

The following theorem will be of basic importance in our reasoning:

THEOREM. There exists a uniquely determined function $p_{h k}$ in $C_{\lambda}$ which minimizes $m(W, p)$,

$$
\min _{p \in C_{\lambda}} m(W, p)=m\left(W, p_{h k}\right) .
$$

For various $h, k$, the functions $p_{h k}$ are interrelated by

$$
p_{h k}=h p_{0}+k p_{1},
$$

where $p_{0}, p_{1}$ stand for $p_{1,0}, p_{0,1}$, respectively.

The minimum value of $m(W, p)$ can be expressed solely in terms of the $a_{\nu}$-coefficients of $p_{0}, p_{1}$ :

$$
m\left(W, p_{h k}\right)=k^{2} \mu_{1}-h^{2} \mu_{0},
$$

$\mu_{0}, \mu_{1}$ being the values of $\mu$ for $p_{0}, p_{1}$. The deviation from this minimum is given by the Dirichlet integral:

$$
m(W, p)=k^{2} \mu_{1}-h^{2} \mu_{0}+D\left(p-p_{h k}\right) .
$$

The proof, based on the reduction theorem, will be given in nos. 8-17.

8. We first have to show that condition $1^{\circ}$ of the reduction theorem is satisfied, that is, the problem can be solved for a Riemann surface $W$ whose boundary $\beta$ consists of a finite, number of analytic Jordan curves.

In this case there exist in $C_{1}$ two uniquely determined functions $p_{0}, p_{1}$, 
defined by the following properties:

$$
\left\{\begin{aligned}
\partial p_{0} / \partial n & =0 \text { on } \beta, \\
p_{1} & =\text { const. on } \beta .
\end{aligned}\right.
$$

These functions can be easily constructed by the linear operator method [16], for the basic condition $\int d s^{*}=0$ is obviously satisfied for both $p_{0}$ and $p_{1}$. This implies that the functions in the sequel can be formed purely constructively.

We set

$$
p_{h k}=h p_{0}+k p_{1},
$$

and shall show that the deviation formula (7) holds. To begin with, we have

$$
D\left(p-p_{h k}\right)=\int_{\beta} p d p^{*}+\int_{\beta} p_{h k} d p_{h k}^{*}-\int_{\beta} p d p_{h k}^{*}-\int_{\beta} p_{h k} d p^{*} .
$$

9. The second integral on the right simplifies immediately:

$$
\int_{\beta} p_{h k} d p_{h k}^{*}=h k \int_{\beta} p_{0} d p_{1}^{*}=h k \int_{\beta} p_{0} d p_{1}^{*}-p_{1} d p_{0}^{*} .
$$

By Green's formula, the last integral can be transferred to the peripheries $\alpha_{i}$ of the $K_{i}$ :

$$
\int_{\beta} p_{h k} d p_{h k}^{*}=h k \sum_{i} \int_{\alpha_{i}} p_{0} d p_{1}^{*}-p_{1} d p_{0}^{*}
$$

If we set $p_{q}=u_{q}+s_{q}(q=0,1)$ in $K_{i}$, with

$$
\begin{aligned}
& u_{q}=\operatorname{Re}\left\{\sum_{0}^{\infty} a_{q \nu} z^{\nu}+\sum_{1}^{m} b_{\nu} z^{-\nu}\right\}, \\
& s_{q}=c \log (1 /|z|),
\end{aligned}
$$

then

$$
\int_{\alpha_{i}} p_{0} d p_{1}^{*}-p_{1} d p_{0}^{*}=\int_{\alpha_{i}} u_{0} d u_{1}^{*}-u_{1} d u_{0}^{*}+\int_{\alpha_{i}}\left(u_{0}-u_{1}\right) d s_{q}^{*} .
$$

As $U_{0}=u_{0}+i u_{0}^{*}$ and $U_{1}=u_{1}+i u_{1}^{*}$ are single-valued in $K_{i}$, one obtains, on integrating by parts,

$$
\int_{\alpha_{i}} u_{0} d u_{1}^{*}-u_{1} d u_{0}^{*}=\int_{\alpha_{i}} u_{0} d u_{1}^{*}+u_{0} d u_{1}^{*}=\operatorname{Im} \int_{\alpha_{i}} U_{0} d U_{1}
$$

The integral here can be written 


$$
\begin{aligned}
\int_{\alpha_{i}} U_{0} d U_{1} & =\int_{\alpha_{i}}\left(\sum_{0} a_{0 \nu} z^{\nu}+\sum_{1} b_{\nu} z^{-\nu}\right)\left(\sum_{1} \nu a_{1 \nu} z^{\nu-1}-\sum_{1} \nu b_{\nu} z^{-\nu-1}\right) d z \\
& =\int_{\alpha_{i}}\left(-\sum_{1} \nu b_{\nu} a_{0 \nu}+\sum_{1} \nu b_{\nu} a_{1 \nu}\right) \frac{d z}{z} \\
& =2 \pi i \sum_{1} \nu \dot{b}_{\nu}\left(a_{1 \nu}-a_{0 \nu}\right) .
\end{aligned}
$$

The latter part of (12) has the value

$$
\int_{\alpha_{i}}\left(u_{0}-u_{1}\right) d s_{q}^{*}=-c \int_{\alpha_{i}}\left(u_{0}-u_{1}\right) d \arg z=2 \pi \operatorname{Re}\left\{c\left(a_{10}-a_{00}\right)\right\},
$$

and we find, on combining (11)-(15), that

$$
\begin{aligned}
\int_{\beta} p_{h k} d p_{h k}^{*} & =2 \pi h k \sum_{i} \operatorname{Re}\left\{c\left(a_{10}-a_{00}\right)+\sum_{1} \nu b_{\nu}\left(a_{1 \nu}-a_{0 \nu}\right)\right\} \\
& =h k\left(\mu_{1}-\mu_{0}\right) .
\end{aligned}
$$

10. For the third integral in (10), we have similarly

$$
\int_{\beta} p d p_{h k}^{*}=k \int_{\beta} p d p_{1}^{*}-p_{1} d p^{*}=k \sum_{i} \int_{\alpha_{i}} p d p_{1}^{*}-p_{1} d p^{*} .
$$

On setting $p=u+s$ with

$$
\begin{aligned}
& u=\operatorname{Re}\left\{\sum_{0}^{\infty} a_{\nu} z^{\nu}+(h+k) \sum_{1}^{m} b_{\nu} z^{-\nu}\right\}, \\
& s=(h+k) c \log (1 /|z|),
\end{aligned}
$$

one obtains

$$
\int_{\alpha_{i}} p d p_{1}^{*}-p_{1} d p^{*}=\int_{\alpha_{i}} u d u_{1}^{*}-u_{1} d u^{*}+\int_{\alpha_{i}} u d s_{1}^{*}-u_{1} d_{s}^{*} .
$$

Here

$$
\int_{\alpha_{i}} u d u_{1}^{*}-u_{1} d u^{*}=\operatorname{Im} \int_{\alpha_{i}} U d U_{1}
$$

with

$$
\begin{aligned}
\int_{\alpha_{i}} U d U_{1} & =\int_{\alpha_{i}}\left[\sum_{0} a_{\nu} z^{\nu}+(h+k) \sum_{1} b_{\nu} z^{-\nu}\right]\left[\sum_{1} \nu a_{1 \nu} z^{\nu-1}-\sum_{1} \nu b_{\nu} z^{-\nu-1}\right] d z \\
& =2 \pi i \sum_{1} \nu b_{\nu}\left[(h+k) a_{1 \nu}-a_{\nu}\right],
\end{aligned}
$$

and 


$$
\int_{\alpha_{i}} u d s_{1}^{*}-u_{1} d s^{*}=-2 \pi \operatorname{Re}\left\{c a_{0}-(h+k) c a_{10}\right\} .
$$

Consequently,

$$
\begin{aligned}
\int_{\beta} p d p_{h k}^{*} & =2 \pi k \sum_{i} \operatorname{Re}\left\{c\left[(h+k) a_{10}-a_{0}\right]+\sum_{1} \nu b_{\nu}\left[(h+k) a_{1 v}-a_{\nu}\right]\right\} \\
& =k\left[(h+k) \mu_{1}-\mu\right] .
\end{aligned}
$$

11. Finally, for the last term in (10), we have

$$
\begin{aligned}
\int_{\beta} p_{h k} d p^{*} & =h \int_{\beta} p_{0} d p^{*}-p d p_{0}^{*}=h \sum_{i} \int_{\alpha_{i}} p_{0} d p^{*}-p d p_{0}^{*} \\
& =h \sum_{i}\left(\int_{\alpha_{i}} u_{0} d u^{*}-u d u_{0}^{*}+\int_{\alpha_{i}} u_{0} d s^{*}-u d s_{0}^{*}\right) .
\end{aligned}
$$

This gives, as in no. 10 ,

$$
\begin{aligned}
\int_{\beta} p_{h k} d p^{*} & =2 \pi h \sum_{i} \operatorname{Re}\left\{c\left[a_{0}-(h+k) a_{00}\right]+\sum_{1} \nu b_{\nu}\left[a_{\nu}-(h+k) a_{0 \nu}\right]\right\} \\
& =h\left[\mu-(h+k) \mu_{0}\right] .
\end{aligned}
$$

12. On summing up, we find from (10), (16), (17), (18) that

$$
\int_{\beta} p d p^{*}+(k-h) \mu=\left(k^{2} \mu_{1}-h^{2} \mu_{0}\right)+D\left(p-p_{h k}\right) .
$$

Since $D\left(p-p_{h k}\right)$ is nonnegative and vanishes for $p=p_{h k}$, we conclude that the functional on the left is minimized by $p_{h k}$, and the value of the minimum is $k^{2} \mu_{1}-h^{2} \mu_{0}$. Thus, condition $1^{\circ}$ of the reduction theorem is fulfilled and the deviation formula (7) holds in the special case where $\beta$ consists of a finite number of analytic Jordan curves.

13. Condition $2^{\circ}$ of the reduction theorem is easily verified. In fact, for every $p \in C_{n+1}$,

$$
m\left(W_{n+1}, p\right)-m\left(W_{n}, p\right)=\int_{\beta_{n+1}-\beta_{n}} p d p^{*},
$$

where the integral equals the Dirichlet integral of $p$ over $W_{n+1}-W_{n}$, thus being nonnegative.

14. To prove that condition $3^{\circ}$ of the reduction theorem is satisfied, let now $W$ be an arbitrary open Riemann surface, with an exhaustion $\left\{W_{n}\right\}$ by compact subregions $W_{n}$, bounded by finite sets $\beta_{n}$ of analytic Jordan curves. Let $p^{n}$ be the function $p_{h k}=h p_{0}+k p_{1}$ for $W_{n}$. More precisely, $p^{n}$ minimizes the functional 


$$
m\left(W_{n}, p\right)=\int_{\beta_{n}} p d p^{*}+(k-h) \mu
$$

in the class $C_{n}$ of functions $p$ harmonic on $W_{n}$ except at the singularities $\zeta_{i}$. Correspondingly, we write $p_{0}^{n}, p_{1}^{n}, \mu_{0}^{n}, \mu_{1}^{n}$ for $p_{0}, p_{1}, \mu_{0}, \mu_{1}$ on $W_{n}$. If $D_{N}$ denotes the Dirichlet integral over a fixed $W_{N}$, then, by (19) for $W_{N}$, and for $n>N$,

$$
\begin{aligned}
D_{N}\left(p^{n}-p^{N}\right) & =m\left(W_{N}, p^{n}\right)-m\left(W_{N}, p^{N}\right) \\
& \leqq m\left(W_{n}, p^{n}\right)-m\left(W_{N}, p^{N}\right) \\
& =k^{2}\left(\mu_{1}^{n}-\mu_{1}^{N}\right)-h^{2}\left(\mu_{0}^{n}-\mu_{0}^{N}\right) .
\end{aligned}
$$

On setting $h=1, k=0$ and then $h=0, k=1$ in (19) for $W_{N}$, we infer that $\mu_{1}^{N} \leqq \mu \leqq \mu_{0}^{N}$ for functions $p$ with $\int_{\beta_{N}} p d p^{*} \leqq 0$. The latter condition is fulfilled, in particular, by $p_{0}^{n}, p_{1}^{n}$, for $\int_{\beta_{N}} p_{0}^{n} d p_{0}^{n *} \leqq \int_{\beta_{n}} p_{0}^{n} d p_{0}^{n *}=0$, and the same is true of $p_{1}^{n}$. Consequently,

$$
D_{N}\left(p^{n}-p^{N}\right) \leqq\left(h^{2}+k^{2}\right)\left(\mu_{0}^{N}-\stackrel{N}{\mu_{1}}\right) .
$$

Here the right-hand side is independent of $n$, and we conclude that the family $p^{n}$ is normal. Thus condition $3^{\circ}$ of the reduction theorem is fulfilled.

15. The reduction theorem now provides us with the existence on $W$ of a limiting function $p_{h k}$ of a subsequence, say again $\left\{p^{n}\right\}$, with the property

$$
\min _{p \in C_{\lambda}} m(W, p)=m\left(W, p_{h k}\right) \text {. }
$$

Moreover, the value of this minimum is

$$
m\left(W, p_{h k}\right)=k^{2} \mu_{1}-h^{2} \mu_{0},
$$

where $\mu_{1}, \mu_{0}$ are the limits of $\mu_{1}^{n}, \mu_{0}^{n}$.

16. The deviation formula will now be based on the minimum property of $p_{h k}$. If we set $p-p_{h k}=u$ for $p \in C_{\lambda}$, then, for real $\epsilon$,

$$
\begin{aligned}
m\left(W_{n}, p_{h k}+\epsilon u\right)= & m\left(W_{n}, p_{h k}\right)+\epsilon^{2} D_{n}(u) \\
& +\epsilon\left[\int_{\beta_{n}}\left(p_{h k} d u^{*}+u d p_{h k}^{*}\right)+(k-h)\left(\mu-\mu_{h k}\right)\right] .
\end{aligned}
$$

Suppose first that $D(u)<\infty$ over $W$. Then the first three terms of this formula tend to finite limits for $n \rightarrow \infty$, and so does, a fortiori, the expression $I_{n}$, say, in the brackets; set $I=\lim I_{n}$. It follows that

$$
m(W, p)_{h k}+\epsilon u=m\left(W, p_{h k}\right)+\epsilon^{2} D(u)+\epsilon I .
$$

By the minimum property, $d m / d \epsilon=0$ for $\epsilon=0$, whence $I=0$. On setting $\epsilon=1$ we have the desired deviation formula

$$
m\left(W, p_{h k}+\epsilon u\right)=m\left(W, p_{h k}\right)+D\left(p-p_{h k}\right) .
$$


This holds, trivially, in the case $D\left(p-p_{h k}\right)=\infty$ as well. In fact, if $W_{0}$ is a compact subregion containing in its interior the singularities $\zeta_{i}$ of $p$, then the Dirichlet integrals $D^{0}$ extended over $W-W_{0}$ satisfy the triangle inequality

$$
\left(D^{0}\left(p-p_{h k}\right)\right)^{1 / 2} \leqq\left(D^{0}(p)\right)^{1 / 2}+\left(D^{0}\left(p_{h k}\right)\right)^{1 / 2} .
$$

Since, by (16) for $W, \int_{\beta} p_{h k} d p_{h k}^{*}$ is always finite, so is $D^{0}\left(p_{h k}\right)$, while the finiteness of $m(W, p)$ would imply that of $\int_{\beta} p d p^{*}$ and of $D^{0}(p)$. But then $D\left(p-p_{h k}\right)$ would be finite, contrary to the assumption. We infer that $m(W, p)$ must be infinite, and the deviation formula in this degenerate form continues to hold.

17. It remains to show that the minimizing function $p_{h k}$ is unique. Suppose $p^{\prime}, p^{\prime \prime}$ are two minimizing functions. Then

$$
D\left(p^{\prime}-p^{\prime \prime}\right)=m\left(W, p^{\prime}\right)-m\left(W, p^{\prime \prime}\right)=0
$$

and $p^{\prime}-p^{\prime \prime}$ is constant. By the normalization $\operatorname{Re}\left\{a_{0}\right\}=0$ at the center $\zeta_{1}$ of $K_{1}$, this constant vanishes, and we have $p^{\prime} \equiv p^{\prime \prime}$. The proof of our theorem (no. 7) is herewith complete.

\section{§3. INTERPOLATION OF HARMONIC FUNCTIONS}

18. We now turn to the following

INTER POLATION PROBLEM. Given an open Riemann surface $W$, and a finite number of points $\zeta_{i}(i=1, \cdots, n)$ on $W$. Minimize the Dirichlet integral $D(u)$ among all harmonic functions $u(z), z=x+i y$, on $W$ with prescribed values, $u_{i \nu}$, of $\partial^{\nu} u\left(\zeta_{i}\right) / \partial x^{\nu}, \nu=0, \cdots, m$.

Again we may choose the points $\zeta_{i}$ to be the centers of disjoint parametric disks $K_{i}$. Then the $u(z)$ have the expansions

$$
u(z)=\operatorname{Re}\left\{\sum_{\nu=0}^{\infty} a_{\nu}^{i} z\right\}
$$

the indices $i$ now being written down to indicate the individual parametric disks. In terms of these expansions, the prescribed quantities are

$$
\operatorname{Re}\left\{a_{\nu}^{i}\right\}=u_{i \nu}
$$

19. The theorem of no. 7 has the following immediate consequence:

The function $u_{0}=p_{0}-p_{1}$ gives to the Dirichlet integral the minimum

$$
\min D(u)=\mu_{0}-\mu_{1}
$$

among all harmonic functions $u$ on $W$ with

$$
\mu=\mu_{0}-\mu_{1} .
$$

The deviation from this minimum is measured by

$$
D\left(u-u_{0}\right)=D(u)-D\left(u_{0}\right) .
$$

In fact, the class determined by (3) is certainly not empty, for $u_{0}$ belongs 
to it. The functions $p \in C_{0}(\lambda=0)$ are regular harmonic, and in the case $h=1, k=-1$, formula $(7)$, no. 7 , can be written

$$
D(u)-2 \mu=\mu_{1}-\mu_{0}+D\left(u-u_{0}\right) .
$$

For $\mu=\mu_{0}-\mu_{1}$, the statement follows.

20. Condition (3) is fulfilled, in particular, by the functions $u$ with

$$
\operatorname{Re}\left\{a_{\nu}^{i}\right\}=\operatorname{Re}\left\{a_{0 \nu}^{i}-a_{1 \nu}^{i}\right\},
$$

$\nu=0, \cdots, m$, if the $b_{\nu}$ are taken real. Thus we have the solution of our interpolation problem for the special values on the right. If these values can be arbitrarily prescribed, by properly choosing the $b_{\nu}^{i}$ and $c^{i}$ in the expansions

$$
p=\operatorname{Re}\left\{\sum_{0}^{\infty} a_{\nu}^{i} z^{\nu}+\lambda \cdot\left(\sum_{1}^{m} b_{\nu}^{i-\nu}-c^{i} \log |z|\right)\right\},
$$

then the solution of our problem is complete. Our task amounts to studying when such a choice is possible.

21. The functions $p_{0}, p_{1}$ can be expressed as linear combinations of certain "elementary functions". To see this, we choose the $b_{\nu}^{i}=0, c^{1}=-1, c^{i}=1$ ( $j$ fixed $\neq 1$ ), the other $c^{i}=0$. Then we have a class $\left\{s^{i}\right\}$ of harmonic functions with two logarithmic poles only; let $s_{0}^{j}, s_{1}^{j}$ be the functions $p_{0}, p_{1}$ in this special class. Similarly, take the $c^{i}=0, b_{k}^{j}=1\left(j, k\right.$ fixed), the other $b_{\nu}^{i}=0$. Now we have a single pole at a fixed $\zeta_{j}$, of order $k(\leqq m)$, and we denote the corresponding functions $p_{0}, p_{1}$ by $t_{\mathbf{k} 0}^{j}, t_{\mathbf{k} 1}^{j}$.

In the general class $C_{\lambda}$ with arbitrary real $b_{\nu}^{i}, c^{i}$ the functions $p_{0}, p_{1}$ can be written

$$
\begin{aligned}
& p_{0}=\sum_{j=2}^{n} c^{j} s_{0}^{j}+\sum_{j=1}^{n} \sum_{k=1}^{m} b_{k}^{j} t_{k 0}^{j}, \\
& p_{1}=\sum_{j=2}^{n} c^{j} s_{1}^{j}+\sum_{j=1}^{n} \sum_{k=1}^{m} b_{k}^{j} t_{k 1}^{j} .
\end{aligned}
$$

Indeed, for an approximating region $W_{n}$, the functions $p_{0}, p_{1}$ are uniquely determined in $C_{1}$ by the boundary conditions $\partial p_{0} / \partial n=0, p_{1}=$ const. on $\beta_{n}$. As these conditions are fulfilled by the $s_{0}^{j}, s_{1}^{j}$ and $t_{k 0}^{j}, t_{k 1}^{j}$, so they are by the linear combinations (7) which therefore constitute $p_{0}, p_{1}$. For $W_{n} \rightarrow W$, all these functions converge to unique limiting functions, and the relations (7) remain valid on $W$.

22. The difference

$$
p_{0}-p_{1}=\sum_{j=2}^{n} c^{j}\left(s_{0}^{j}-s_{1}^{j}\right)+\sum_{j=1}^{n} \sum_{k=1}^{m} b_{k}^{j}\left(t_{k 0}^{j}-t_{k 1}^{j}\right)
$$

has the expansion 


$$
p_{0}-p_{1}=\operatorname{Re}\left\{\sum_{\nu=0}^{\infty}\left(a_{0 \nu}^{i}-a_{1 v}^{i}\right) z^{\nu}\right\}
$$

in $K_{i}$. For the $s$-functions we write in $K_{i}$

$$
s_{0}^{j}-s_{1}^{j}=\operatorname{Re}\left\{\sum_{\nu=0}^{\infty} \sigma_{\nu}^{j i} z\right\}
$$

with $j=2, \cdots, n ; i=1, \cdots, n$. For the $t$-functions we set, correspondingly,

$$
t_{k 0}^{j}-t_{k 1}^{j}=\operatorname{Re}\left\{\sum_{v=0}^{\infty} \tau_{k v}^{j i} z\right\}
$$

Then, by (8),

$$
\operatorname{Re}\left\{\sum_{j=2}^{n} c^{j} \sigma_{\nu}^{j i}+\sum_{j=1}^{n} \sum_{k=1}^{m} b_{k}^{j} \tau_{k \nu}^{j i}\right\}=\operatorname{Re}\left\{a_{0 \nu}^{i}-a_{1 \nu}^{i}\right\}
$$

for $i=1, \cdots, n ; \nu=0, \cdots, m$.

23. In the relations (12), the $\sigma$ 's and $\tau$ 's are known constants, and the unknown real constants $c$ 's and $b$ 's are to be so determined that the right-hand sides assume the prescribed values $u_{i v}$. By the normalization $R\left\{a_{0}^{1}\right\}=0$ for all $p, s, t$, no equation corresponds to $i=1, \nu=0$. Thus we are dealing with $n-1+n m$ equations with the same number of unknowns. A solution exists if and only if the determinant

$$
\Delta=\left|\operatorname{Re}\left\{\sigma_{\nu}^{j i}\right\}, \operatorname{Re}\left\{\tau_{k \nu}^{j i}\right\}\right|
$$

is different from zero. Here the rows are formed for varying $j, k$, the columns for $i, \nu$.

24. We have thus arrived at the solution of our interpolation problem:

Theorem. Given an arbitrary Riemann surface $W$, and points $\zeta_{i}$ $(i=1, \cdots, n)$ on $W$, let $u_{i \nu}(\nu=0, \cdots, m)$ be arbitrary finite real constants. Let $\{u\}$ be the class of regular single-valued harmonic functions on $W$ with

$$
\partial^{\nu} u\left(\zeta_{i}\right) / \partial x^{\nu}=u_{i v}
$$

There exists, in $\{u\}$, a function $u_{0}$ which minimizes the Dirichlet integral,

$$
\min D(u)=D\left(u_{0}\right) \text {, }
$$

if and only if, in (13), $\Delta \neq 0$.

The minimizing function is unique and has the expression

$$
u_{0}=p_{0}-p_{1},
$$

where $p_{0}, p_{1}$ are taken in that class $C_{1}$ whose coefficients $b_{\nu}^{t}, c^{i}$ give the prescribed values $u_{i v}$ to the expressions (12).

In terms of these coefficients, the minimum of $D(u)$ is $\mu_{0}-\mu_{1}$, and the devia- 
tion from this minimum is measured by the Dirichlet integral of $u-u_{0}$ :

$$
D(u)=\mu_{0}-\mu_{1}+D\left(u-u_{0}\right) .
$$

The remark might be added that the above reasoning remains valid verbatim in the case where the values of $u\left(\zeta_{i}\right)$ and $U^{(\nu)}\left(\zeta_{i}\right), \nu=1, \cdots, m$, are prescribed, with $u=\operatorname{Re}\{U\}$.

\section{§4. INTERPOLATION OF ANALYTIC FUNCTIONS}

25. In what follows, $W$ signifies a planar Riemann surface. We consider single-valued analytic functions $Q=q+i q^{*}$ on $W$, with expansions

$$
Q=\sum_{0}^{\infty} a_{\nu}^{i \nu} z^{\nu}+\lambda \cdot \sum_{1}^{m} b_{\nu}^{i-\nu}
$$

about the $\zeta_{i}$. For fixed complex $b_{\nu}^{i}$ and subsequently fixed real $\lambda$, the class of functions $Q$ with normalization $a_{0}^{1}=0$ shall be designated by $G_{\lambda}$. The symbol $\mu$ now stands for

$$
\mu=2 \pi \operatorname{Re} \sum_{i=1}^{n} \sum_{\nu=1}^{m} \nu b_{\nu}^{i} a_{\nu}^{i} .
$$

If $W$ is compact and its boundary $\beta$ consists of a finite number of analytic Jordan curves $\gamma$, there are unique functions $Q_{0}, Q_{1}$ in $G_{1}$, determined by the conditions

$$
\begin{aligned}
\partial q_{0} / \partial n & =0 \text { on } \beta, \\
q_{1} & =r_{\gamma} \text { on } \gamma .
\end{aligned}
$$

The constants $r_{\gamma}$ are so chosen that

$$
\int_{\gamma} d q_{1}^{*}=0
$$

along each contour $\gamma \subset \beta$.

For an open $W, q_{0}, q_{1}$ appear as limiting functions of the $q_{0}, q_{1}$ for exhausting subregions $W_{n}$. The reasoning in $\S \S 2,3$ remains valid throughout when $p, p_{0}, p_{1}$ are replaced by $q, q_{0}, q_{1}$, and the basic formula now takes the form

$$
\int_{\beta} q d q^{*}+(k-h) \mu=k^{2} \mu_{1}-h^{2} \mu_{0}+D\left(q-q_{h k}\right) .
$$

26. For regular single-valued analytic functions $V$ on $W$ with expansions

$$
V=\sum_{\nu=0}^{\infty} a_{\nu}^{i v}
$$

in $K_{i}$ and with $\mu=\mu_{0}-\mu_{1}$, we conclude from (5): 
The function $V_{0}=Q_{0}-Q_{1}$ has the minimum property

$$
D(V)=\mu_{0}-\mu_{1}+D\left(V-V_{0}\right) .
$$

The condition $\mu=\mu_{0}-\mu_{1}$ is satisfied, in particular, by those functions for which

$$
a_{v}^{i}=\stackrel{i}{i} a_{0 v}-\stackrel{i}{a_{1 v}},
$$

$\nu=1, \cdots, m$. If here the right sides can be prescribed at will, by properly choosing the complex $b_{\nu}^{i}$, then we are in possession of a function $V$ with arbitrarily given values of the $V^{(\nu)}\left(\zeta_{i}\right)$, and with a minimal $D(V)$.

27. In analogy to no. 21 , let $Q_{k 0}^{j}, Q_{k 1}^{j}$ be the functions $Q_{0}, Q_{1}$ with the sole singularity $1 / z^{k}$ at $\zeta_{j}$. For the corresponding functions $Q_{k 0}^{j \phi}, Q_{k 1}^{j \phi}$ with poles $e^{i \phi} / z^{k}$ at $\zeta_{j}$, we have

$$
\begin{aligned}
& Q_{k 0}^{j \phi}=Q_{k 0}^{j} \cos \phi+i Q_{k 1}^{j} \sin \phi, \\
& Q_{k 1}^{j \phi}=i Q_{k 0}^{j} \sin \phi+Q_{k 1}^{j} \cos \phi .
\end{aligned}
$$

In fact, on a compact $W$ bounded by analytic Jordan curves, the combinations on the right have the same singularity as $Q_{k 0}^{j \phi}, Q_{k 1}^{j \phi}$. Moreover, the real part of the first combination has vanishing normal derivative on the boundary, while that of the second is constant on each contour. For an open $W$, the relations (9) then follow by an exhaustion $W_{n} \rightarrow W$.

We obtain from (9),

$$
Q_{k 0}^{j \phi}-Q_{k 1}^{j \phi}=e^{-i \phi}\left(Q_{k 0}^{j}-Q_{k 1}^{j}\right)
$$

and conclude that (8), no. 22 , is now replaced by

$$
Q_{0}-Q_{1}=\sum_{j=1}^{n} \sum_{k=1}^{m} \overline{b_{k}^{j}}\left(Q_{k 0}^{j}-Q_{k 1}^{j}\right)
$$

the bar indicating the complex conjugate. Equation (12), no. 22, takes the form

$$
\sum_{j=1}^{n} \sum_{k=1}^{m} \overline{b_{k}^{j} \tau_{k \nu}^{j i}}=a_{0 \nu}^{i}-a_{1 \nu}^{i}
$$

For prescribed right sides, we now have $n m$ equations for the same number of unknowns $b_{j}^{k}$.

28. We proceed to demonstrate that, in the present case, the solvability of the system is characterized by the existence of functions $A D$ on $W$, that is, single-valued nonconstant analytic functions with a finite-Dirichlet integral. The class of Riemann surfaces without functions $A D$ is said to have an $A D$-removable boundary and is denoted by $O_{A D}$. The necessity of the condition $W \notin O_{A D}$ is obvious, for we must have functions on $W$ whose Dirichlet integral could be minimized. 
To establish the sufficiency, suppose that there is indeed a function $A D$ on $W$. Then $W$ can be mapped onto a plane region which contains the point at infinity and whose complement has a positive area (Ahlfors-Beurling [2]). Let $w(z)=u+i v$ be the mapping function, and denote the images of $W, \beta, \zeta_{i}$ by $W, \beta, w_{i}$, the complement of $W$, by $H$. By (7), we have on setting $h=k=1$ in $(5)$,

$$
D\left(Q_{0}-Q_{1}\right)=\mu_{0}-\mu_{1}=-\int_{\beta}\left(q_{0}+q_{1}\right) d\left(q_{0}+q_{1}\right)^{*}=\max _{G_{2}} \int_{-\beta} q d q^{*} .
$$

The function

$$
Q(w)=2 \sum_{i=1}^{n} \sum_{\nu=1}^{m} \tilde{b}_{k}^{j}\left(w-w_{i}\right)^{->}
$$

belongs to $G_{2}$ if the $\tilde{b}_{k}^{j}$ are so chosen that $Q(w(z))$ has the given coefficients $2 b_{k}^{j}$ at the $\zeta_{i}$. Since $Q(w)$ is nonconstant, its Dirichlet integral over $H$ does not vanish,

$$
D_{H}(Q)=\int_{-\beta} q d q^{*}>0,
$$

and it follows that $D\left(Q_{0}-Q_{1}\right)>0$. From this we infer that $Q_{0}-Q_{1}$ and, a fortiori, the linear combination on the right in (11) is not constant. Consequently, the functions $Q_{k 0}^{j}-Q_{k 1}^{j}$ are linearly independent; the system (12) has a solution for any prescribed values on the right.

29. We have established the following result:

Theorem. On a planar Riemann surface $W$, let $\{V\}$ be the class of regular single-valued analytic functions with arbitrarily prescribed values

$$
d^{(\nu)} V\left(\zeta_{i}\right) / d z^{\nu}=V_{i v}
$$

at given points $\zeta_{i}, i=1, \cdots, n ; \nu=1, \cdots, m$. There exists a function $V_{0}$ in $\{V\}$ with the property

$$
\min _{\{V\}} D(V)=D\left(V_{0}\right)
$$

if and only if $W \notin O_{A D}$.

The minimizing function is

$$
V_{0}=Q_{0}-Q_{1}
$$

where $Q_{0}, Q_{1}$ are taken in the class $G_{1}$ determined by the coeffcients $b_{\nu}^{i}$ which give the values $V_{i v}$ to the quantities (12). The value of the minimum and the deviation from the minimum are given, in terms of these coefficients, by the formula

$$
D(V)=\mu_{0}-\mu_{1}+D\left(V-V_{0}\right) \text {. }
$$




\section{LITERATURE}

1. L. Ahlfors, Open Riemann surfaces and extremal problems on compact subregions, Comment. Math. Helv. vol. 24 (1950) pp. 100-134.

2. L. Ahlfors and A. Beurling, Conformal invariants and function-theoretic null-sets, Acta Math. vol. 83 (1950) pp. 101-129.

3. S. Bergman, The kernel function and conformal mapping, Mathematical Surveys, No. 5, New York, American Mathematical Society, 1950.

4. P. Garabedian and M. Schiffer, Identities in the theory of conformal mapping, Trans. Amer. Math. Soc. vol. 65 (1949) pp. 187-238.

5. H. Grunsky, Neue Abschätzungen zur konformen Abbildungen ein- und mehrfach zusammenhängender Bereiche, Schr. Math. Semin. Univ. Berlin vol. 1 (1932) pp. 95-140.

6. T. Kubota, Ueber konforme Abbildungen I, The Science Reports of Tôhoku Imperial University ser. I vol. 9 (1920).

7. - Ueber konforme Abbildungen II, ibid. vol. 10 (1921).

8. O. Lehto, Anwendung orthogonaler Systeme auf gewisse funktionen-theoretische Extremalund Abbildungsprobleme, Annales Academiae Scientiarum Fennicae, Series A. I. no. 59 (1949) $51 \mathrm{pp}$.

9. O. Lokki, Ueber analytische Funktionen, deren Dirichletintegral endlich ist und die in gegebenen Punkten vorgeschriebene Werte annehmen, ibid. no. 39 (1947) 57 pp.

10. - Beiträge zur Theorie der analytischen und harmonischen Funktionen mit endlichem Dirichletintegral, ibid. no. 92 (1951) 11 pp.

11. Ueber reguläre harmonische Funktionen mit endlichem Dirichletintegral, XI Congr. Scand. Math., Trondheim, 1949, Johan Grundt Tanums Förlag, Oslo (1952) pp. 239-242.

12. - Ueber eindeutige analytische Funktionen mit endlichem Dirichlet-integral, Annales Academiae Scientiarum Fennicae, Series A. I. no. 105 (1951) 13 pp.

13. R. Nevanlinna, Ueber beschränkte Funktionen die in gegebenen Punkten vorgeschriebene Werte annehmen, ibid. Series A. XIII. (1919) 71 pp.

14. - - Ueber beschränkte analytische Funktionen, Comm. in honorem Ernesti Leonardi Lindelöf, Helsinki, 1929, 75 pp.

15. G. Pick, Ueber die Beschränkungen analytischer Funktionen, welche durch vorgegebene Funktionenwerte bewirkt werden, Math. Ann. vol. 77 (1916) pp. 7-23.

16. L. Sario, A linear operator method on arbitrary Riemann surfaces, Trans. Amer. Math. Soc. vol. 72 (1952) pp. 281-295.

17. - An extremal method on arbitrary Riemann surfaces, Trans. Amer. Math. Soc. vol. 73 (1952) pp. 459-470.

18. - Minimizing operators on subregions, Proc. Amer. Math. Soc. vol. 4 (1953) pp. 350-355.

19. - Functionals on Riemann surfaces, Lectures on Functions of a Complex Variable, Ann Arbor, 1953 (to appear in 1955).

20. S. Takenaka, General mean modulus of analytic functions, Tôhoku Math. J. vol. 27 (1926) pp. 21-40.

21. J. L. Walsh, Interpolation and functions analytic interior to the unit circle, Trans. Amer. Math. Soc. vol. 34 (1932) pp. 523-556.

22. H. Weyl, Ueber das Pick-Nevanlinnasche Interpolationsproblem und sein infinitesimales Analogon, Ann. of Math. vol. 36 (1935) pp. 230-254.

HARVARD UNIVERSITY,

Cambridge, Mass. 